\title{
La escuela de Chicago y los enfoques cualitativos: términos y conceptos metodológicos
}

\author{
Juan Ignacio Piovani \\ Universidad Nacional de la Plata. Centro Interdisciplinario de Metodología \\ de las Ciencias Sociales \\ Instituto de Investigaciones en Humanidades y Ciencias Sociales. Consejo Nacional \\ de Investigaciones Científicas y Técnicas \\ jpiovani@unibo.edu.ar
}

Recibido: 01-11-2008

Aceptado: 15-02-2010

\section{Resumen}

Frecuentemente, se atribuye a la Escuela de Chicago un lugar central en el desarrollo de las técnicas cualitativas de investigación social. Por otra parte, se reconoce en la sociología norteamericana que esta escuela ocupó un lugar relativamente hegemónico en la disciplina durante las décadas de 1920 y 1930, valiéndose del American Journal of Sociology como principal instrumento de difusión de sus investigaciones empíricas, líneas teóricas y metodológicas. Pero aquellos que han estudiado la historia de los métodos sociológicos, y en particular los desarrollados por esta escuela, han puesto en cuestión que se trate realmente de métodos cualitativos en el sentido actual. Teniendo esta tensión problemática como marco de referencia, en este trabajo se propone - a partir de un rastreo de los artículos publicados en esa revista en la década de 1920 - indagar los usos y los sentidos que se le atribuían a las expresiones que habitualmente se reconocen como denotativas de un enfoque cualitativo: case study, fieldwork, field research, participant observer y otras afines.

Palabras clave: Escuela de Chicago; American Journal of Sociology; métodos cualitativos.

\begin{abstract}
The Chicago School and the qualitative approaches: methodological terms and concepts
\end{abstract}

The Chicago School of Sociology is usually regarded as crucial in the development of the qualitative techniques of research. Moreover, it is widely accepted that it played a rather hegemonic role in American sociology during the 1920's and 1930's, using the American Journal of Sociology as the main tool for the dissemination of its empirical research, and its theoretical and methodological approaches. However, those who have studied the history of the sociological methods in detail, contest that those developed by the Chicagoans were truly qualitative in the current sense. Taking into account this problematic issue, this article explores, by reviewing articles published in the above-mentioned Journal during the 1920's, the uses and meanings attached to terms usually regarded as denotative of a qualitative approach: case study, fieldwork, field research, participant observer, among others.

Key words: Chicago School; American Journal of Sociology; qualitative methods. 


\begin{tabular}{|c|c|}
\hline \multicolumn{2}{|c|}{ Sumario } \\
\hline Introducción & Sobre el debate cualitativo versus \\
\hline Sobre la importancia de la reflexión & \\
\hline metodológica & Comentarios finales \\
\hline $\begin{array}{l}\text { La relación entre los métodos de las } \\
\text { ciencias sociales y las naturales }\end{array}$ & Referencias bibliográficas \\
\hline
\end{tabular}

\section{Introducción}

En 1892, Albion Small, figura clave en la institucionalización académica de la sociología, organiza, en la Universidad de Chicago, el primer departamento del mundo especializado en esta disciplina ${ }^{1}$. Pocos años después, funda el American Journal of Sociology (1895) — que dirigiría por treinta años—y participa activamente en la constitución de la American Sociological Society (1905), precursora de la actual American Sociological Association.

Las primeras investigaciones empíricas realizadas en el Departamento de Sociología de Chicago eran poco rigurosas y marcadamente moralistas (Cortese, 1995). Cabe recordar al respecto que, en sus inicios, Small confirió al departamento una fuerte impronta cristiana, característica estrechamente vinculada a su biografía personal: al igual que otros de los primeros profesores de la institución, como Henderson y Zueblin, Small era un ministro religioso con un decidido compromiso reformista en el ámbito de lo social.

El trabajo académico comenzó a adquirir connotaciones más profesionales hacia fines de 1910, cuando una importante donación privada le permitió a William I. Thomas - doctorado en el Departamento en 1896 y luego vinculado a éste como docente- iniciar sus investigaciones empíricas sobre inmigración, mucho más articuladas desde el punto vista teórico y sólidas metodológicamente que las de sus predecesores. Esta labor investigativa alcanzaría un punto culminante entre 1918 y 1920 con la publicación de su clásico trabajo - en colaboración con Znaniecki- sobre el campesino polaco en Europa y América, un libro que pronto se convertiría en un icono de la investigación cualitativa.

Pero la Escuela de Chicago toma forma hacia 1920 gracias al trabajo de Robert Park y Ernest Burgess en el campo de la sociología urbana, y una vez que Thomas ya había abandonado el Departamento en medio del escándalo derivado de su arresto por parte del FBI acusado de violar la Mann Act de $1910^{2}$.

Park había llegado al Departamento invitado por Thomas en 1913, y poco

1. En realidad, se trató de un departamento de sociología y antropología hasta que ambas disciplinas se separaron institucionalmente en 1929.

2. La Mann Act o White-Slave Traffic Act era una ley estadounidense que, entre otras cosas, prohibía cruzar fronteras interestatales con mujeres para fines tales como la prostitución. Thomas fue arrestado al cruzar la frontera junto a la señora Granger, una mujer casada con un oficial de las Fuerzas Armadas de EEUU. 
después comenzó a ejercer un notable liderazgo intelectual. Por su parte, Burgess se unió al equipo académico en 1916, iniciando inmediatamente una labor colaborativa con Park en el campo de la investigación y la didáctica ${ }^{3}$. Según Cortese (1995), sería el trabajo de Park —y más adelante también el de Burgess - el que permitiría establecer la hegemonía del Departamento en la sociología norteamericana desde aproximadamente 1915 y hasta mediados de la década de 1930, cuando, luego de la partida de Park, en 1934, comenzó su declinación.

Para muchos, por ejemplo Becker (1999), la Escuela de Chicago constituye simplemente un mito, pero uno tan arraigado que estudios bien documentados, como los de Bulmer (1984) y otros, no han podido desarticular. La idea de escuela suele asociarse, en este caso, con la existencia de un espacio académico en el que «el desarrollo de las ciencias sociales [...] estuvo caracterizado por la fuerte integración social y colegial» (Cortese, 1995: 238). Pero Becker (1999) señala, siguiendo a Platt (1996), que, en realidad, este espacio fue mucho más variado y heterogéneo de lo que habitualmente se supone. A partir de su propia experiencia como estudiante de sociología en Chicago, concluye que allí nunca existió una escuela en el sentido que el término adquiere en los estudios sobre historia del pensamiento. En todo caso, si una escuela y una tradición sociológica típica de Chicago existieron, es en el sentido de «escuela de actividad», concepto propuesto por Gilmore (1988) para referirse a escuelas musicales y retomado por Becker (1999) para describir a la sociología de Chicago: lo que tenían en común sus miembros era el trabajo cooperativo en proyectos prácticos.

Pero, como se decía más arriba, mito o no, la idea de una escuela sociológica en la Universidad de Chicago de los años 1920 ha resultado persistente. Y uno de los aspectos que más han contribuido - y aún contribuyen - para sostener esta idea es el supuesto lugar central que la Escuela tuvo en el desarrollo de los métodos cualitativos de investigación social (Taylor y Bogdan, 1986; Forni, 1993; Vasilachis, 1993; Denzin y Lincoln, 1994). Como sostiene Cortese (1995: 238):

Tal vez la contribución más significativa de los sociólogos de Chicago (muy especialmente Park, Thomas y Burgess) fue el desarrollo de métodos de investigación específicos [...] Algunos de los métodos de investigación más distintivos ligados a la sociología de Chicago son los documentos personales, el trabajo de campo intensivo, las fuentes documentales, el mapeo social y el análisis ecológico.

Sin embargo, aquéllos que han estudiado la historia de los métodos sociológicos, y en particular los desarrollados en este ámbito institucional, han puesto en cuestión que se trate realmente de métodos cualitativos en el sentido

3. Ernest Burgess había estado con anterioridad en la institución en calidad de estudiante y regresó como profesor luego de una estadía en Europa. 
actual. Platt (1982, 1983, 1985), uno de los máximos referentes en el estudio de los aspectos metodológicos de la Escuela de Chicago, ha concluido que el origen del abordaje cualitativo en este ámbito (especialmente a través de su supuesto desarrollo de la observación participante) es sólo «mítico». En la misma línea, podrían considerarse, al menos desde algunos puntos de vista, textos como los de Bryman (2001); Bulmer, (1984); Chapouli (1987); Cressey (1983) y Hammersley (1989).

Teniendo esta tensión problemática como marco de referencia, en este trabajo se propone, a partir de un rastreo de los artículos publicados en el American Journal of Sociology (AJS) durante la década de 1920, indagar los usos y sentidos que se le atribuían a las expresiones que habitualmente se reconocen —en el marco de estudios retrospectivos - como denotativas de un enfoque cualitativo ${ }^{4}$ : case study, fieldwork, field research, participant observer y otras afines ${ }^{5}$.

Se restringe el análisis a las publicaciones de la década de 1920 por varias razones concurrentes. Entre ellas, se destacan las siguientes:

- Se trata de una década que queda centralmente comprendida en el periodo que Cortese (1995) define como hegemónico por parte de la sociología de Chicago (1915-1934).

- Comprende el momento inicial —y uno de los de mayor auge- de la Escuela de Chicago.

- Empieza inmediatamente después de la publicación de The polish peasant in Europe and America, obra paradigmática en el campo de los estudios cualitativos y referencia obligada en los debates metodológicos de la época entre «estudios de caso» (cualitativos) y «métodos estadísticos» (cuantitativos) (véase Lundberg, 1960).

- Coincide, en general, con el período en el que Park y Burgess dictaron su seminario sobre Field studies, clave en la formación metodológica de los sociólogos de Chicago y con la publicación de su obra conjunta Introduction to the Science of Sociology (1921) — conocida como Green Bible—, que para muchos representa el manifiesto de la Escuela, en tanto simboliza una forma particular de entender la disciplina (Cortese, 1995).

- Incluye un momento de intenso debate metodológico en el Departamento de Sociología de Chicago luego de la llegada de Ogburn en 1927 y como consecuencia de su enfática defensa de los métodos estadísticos.

Con el fin de reconstruir los usos y los sentidos aludidos, se constituyó un corpus inicial comprendido por: a) los artículos en cuyo título y/o abstract apa-

4. Los análisis que se presentan en este artículo fueron realizados en colaboración con Eugenia Rausky y Javier Santos, y presentados preliminarmente en el XXVI Congreso de la Asociación Latinoamericana de Sociología, Guadalajara (México), agosto de 2007.

5. Se recurre al análisis de tales términos ya que la expresión «métodos cualitativos» no era corriente en aquella época. Tal como sugiere Lundberg (1960), el concepto al que remite esta última expresión era designado en aquel entonces por medio de los términos que aquí se exploran. 
recen las expresiones señaladas, y $b$ ) las reseñas de libros dedicados a temas metodológicos. En esta selección, se reconocieron distintos tipos de materiales: estudios empíricos, estados de la cuestión (sobre la familia, la delincuencia, la sociología rural, la religiosidad, etc.), análisis focalizados en temas estrictamente metodológicos y, finalmente, reflexiones referidas a cuestiones teóricoepistemólogicas.

El corpus inicial incluyó un total de 46 escritos $\left(18\right.$ artículos $^{6}$ y 28 reseñas de libros). Si bien todos ellos fueron revisados en el marco de la investigación llevada a cabo, en este trabajo se presentan referencias explícitas a 18 publicaciones $^{7}$, poniéndose más énfasis en los artículos —nótese que se citan 13 de los 18 identificados_-, dado que es a partir de su análisis que mejor pueden determinarse los sentidos asociados al uso de los dispositivos metodológicos en cuestión.

Cabe aclarar que se limita el análisis a las publicaciones del AJS por tratarse de una revista emblemática y con larga tradición, fundada y editada por el Departamento de Sociología de Chicago. Sin embargo, no constituía el único ámbito en el que publicaban los miembros de la Escuela de Chicago. Por otra parte, tampoco todos los textos publicados en el AJS en el periodo en cuestión eran de autoría de los profesores e investigadores de la Escuela. Dado que, de hecho, muchos de los textos examinados no son de chicaguenses, una cuestión interesante, y que se retomará más adelante, es si entre éstos se encuentran perspectivas (usos y sentidos de los términos analizados) de mayor cercanía con los principios cualitativos tal como se los entiende habitualmente.

\section{Sobre la importancia de la reflexión metodológica}

Bulmer (1984: 89-90) afirma que, en los artículos de la época, existen pocas definiciones explícitas sobre los métodos de investigación utilizados, y lo atribuye a una cierta falta de conciencia metodológica ${ }^{8}$. Sin embargo, la lectura del corpus da cuenta de una creciente preocupación en este sentido, aunque directamente relacionada con la discusión en torno a la cientificidad de las ciencias sociales y los modos adecuados de definirla (Park, 1923; Boucke, 1923; Nelson, 1924; House, 1926; Hoffer, en Melvin, 1927; Blumer, 1930; Park, 1930).

Park (1923), por ejemplo, destaca la importancia de los métodos en el avance de la ciencia y plantea que la cuestión metodológica de las ciencias sociales era particularmente candente en ese momento. Melvin (1927) subraya que la sociología estaba deviniendo una ciencia y que «en [ese] proceso se ha percibido, y en la actualidad se reconoce ampliamente, la necesidad de

6. Estos 18 artículos incluyen: 12 en los que aparece la expresión case study, 3 en los que se registra el término fieldwork, 2 en los que se utiliza participant observer $\mathrm{y}$, finalmente, 1 en el que se recurre a field research.

7. Véase el anexo para un detalle de estos materiales.

8. Nótese que, para este autor, a diferencia de lo que sostiene Cortese (1995), lo metodológico no era central en la Escuela de Chicago. 
usar métodos científicos». En sus conclusiones, señala que un cuerpo definido de conocimiento metodológico estaba en el proceso de formación. En contraposición, una reseña crítica realizada por Blumer (1930) muestra la confusión que reinaba hacia fines del período estudiado en el campo de las ciencias sociales: para él, lo fundamental de la metodología no sólo no había sido resuelto, sino que no había sido aún debidamente formulado (y comprendido).

Por otra parte, y si bien Bulmer (1984) sostiene acertadamente que existieron muy pocos textos especializados en metodología de la investigación antes de 1930, a partir de la evidencia del material analizado, cabe destacar la presencia creciente de algunos textos — en el formato de manual - que buscaban sistematizar el conocimiento metodológico hasta entonces aún disperso. Estos textos aparecen recurrentemente en el AJS a través de reseñas (Burgess, 1923; Park, 1923; Queen, 1929; Blumer, 1930; Kuhlaman, 1930). Al mismo tiempo, y tal vez evidencia de lo incipiente de la reflexión metodológica, se observa una falta de acuerdo en el uso de los términos específicos, una confusión de niveles de discurso (por ejemplo, entre métodos y técnicas, en el sentido de Marradi, Archenti y Piovani, 2007) o, ya en el campo de las técnicas, entre las de recolección, codificación, análisis, etc. Por ejemplo, reseñando un texto de Crawford, Kuhlaman (1930) señala críticamente el listado de las técnicas de investigación provisto por el autor: experimentales, históricas, psicológicas, de estudio de casos, de confección de currículo, de análisis de trabajo, entrevista, cuestionario, de observación, de medición, estadísticas, tabulares, gráficas y bibliotecnológicas.

\section{La relación entre los métodos de las ciencias sociales y las naturales}

En la formalización del conocimiento metodológico de la época, aparece otra discusión de relevancia: ¿los métodos deberían ser «importados» de las ciencias naturales o desarrollados con un criterio de autonomía?, ¿propuestos a priori o construidos a partir de la investigación empírica? Esto remite nuevamente a la discusión sobre el estatus de la sociología - y de las ciencias sociales en general- - su relación con las ciencias naturales. Para Blumer (1930) el desafío era estudiar críticamente los problemas fundamentales de las ciencias sociales y hacer una derivación empírica de los procedimientos más adecuados. Frente a quienes llamaban a valerse de los métodos de las ciencias naturales (o adaptaciones de ellos) para asegurar el estatus científico de las ciencias sociales, él propone seguir la sugerencia de Dewey: los métodos de las ciencias sociales debían ser indígenas, no importaciones; la metodología debía tomar una nueva orientación y desarrollarse empíricamente. Boucke (1923) apunta, aunque no llega a formularlo con total claridad, el desarrollo de un método específico para las ciencias sociales, al que llama «reflexivo»: una combinación de la inducción — que define como típica de las ciencias naturales - con la deducción formal, que, aunque no dicho en estos términos por el autor, probablemente estaba identificada con corrientes filo- 
sóficas que las ciencias sociales empíricas, y «científicas», estaban llamadas a reemplazar.

Por otra parte, los planteos en torno al desarrollo de metodologías propias están ligados a un cierto consenso acerca de los límites de la experimentación en el campo de las ciencias sociales (Boucke, 1923; Holt, 1926; Hoffer, en Melvin, 1927; Wood, 1927; Zimmermann, en Melvin, 1927; Thomas, 1929; Blumer, 1930). Las respuestas frente a estos límites son de dos tipos: por un lado, desarrollo de alternativas al experimento, pero en el sentido de adaptaciones que cumplieran funciones análogas y respondieran a una misma concepción subyacente de ciencia, de corte positivista (postura fuertemente criticada por Blumer, 1930) y, por otro lado, postulado de metodologías sustantivamente distintas, por fuera de dichos cánones tradicionales.

En el primer grupo, se encuentran los que promovían encendidamente el uso de los métodos estadísticos, por ejemplo Hoffer y Zimmermann (ambos en Melvin, 1927: 206): «el principal acercamiento a la exactitud en el estudio de los fenómenos sociales debe hacerse, no a través del experimento de laboratorio, sino de la estadística» ${ }^{9}$. Ahora bien, con respecto a esto, hay una cuestión interesante a destacar. Para quienes favorecían los métodos estadísticos, su justificación se basaba en la posibilidad de alcanzar objetivos cognitivos análogos a los del experimento, recurriendo con frecuencia al uso de términos clave como objetividad, medición, cuantificación, generalización, ley, etc. ${ }^{10}$. En contraposición, en la actualidad, tenderíamos a pensar que, siempre frente al reconocimiento de los límites del experimento, la promoción de los estudios de caso, la observación participante, las entrevistas, etc. debería inscribirse en la segunda perspectiva, es decir, la de una alternativa más radical, al menos en lo que respecta a los compromisos epistemológicos que el experimento - y los métodos estadísticos-implica. Sin embargo, al examinar los usos de aquellos términos en la literatura de la época, se encuentra que muchos pretendían encuadrar estas propuestas metodológicas bajo los cánones clásicos de las ciencias naturales y, por lo tanto, los promovían como medios aptos para investigar «objetivamente», generalizar, formular leyes, etc. Otros, por cierto muchos menos y en general en un sentido bastante latente, veían en estos métodos alternativas capaces de realizar otro tipo de objetivos en el marco de una concepción diferente de ciencia. Asimismo, sostenían que ellos no podían ni aplicarse ni juzgarse en los términos de la ciencia canónica, acercándose de este modo a algunos de los sentidos más habituales de la investigación social cualitativa contemporánea de corte inter-

9. La cita corresponde a un pasaje escrito por Hoffer en la discusión del artículo de Melvin (1927).

10. Una de las pocas miradas críticas, en que la promoción de estos métodos se complementa con un enfático llamamiento a su aplicación cuidadosa y al examen detallado de lo que pueden y no pueden ofrecer en términos de construcción de conocimiento, así como a la toma de responsabilidad por las consecuencias de su aplicación y a la explicitación de sus bases epistemológicas, se encuentra en el rico artículo de Thomas (1929). 
pretativo. Veamos la lógica de estas dos posturas tomando como ejemplo a los estudios de caso ${ }^{11}$ :

Holt (1926) define el estudio de casos como un método sistemático para acceder a la personalidad humana, las actitudes individuales y los propósitos de vida de los individuos, pero lo defiende en cuanto medio para generalizar y formular leyes.

Chaffee (1930), retomando a Cooley, sostiene las ventajas del estudio de caso para realizar la investigación de pequeños grupos e instituciones. En el caso que presenta, se recupera la historia de la comunidad, su organización política y económica, y se cubren aspectos objetivos y subjetivos. Sin embargo, cree que luego de estudiar varios casos se pueden establecer generalizaciones a partir del control de las conclusiones derivadas de un caso por medio del estudio de otros similares.

Wood (1927), centrado en la investigación criminológica, plantea que, frente a la falta de oportunidad de generar experimentos controlados, se abre la puerta a una variante subjetivista. Dadas las limitaciones de la estadística para revelar la personalidad del criminal, se vuelve necesario el uso de lo que denomina "historias de caso individual y comunitario" como el medio más pertinente para interpretar la conducta, tanto en términos de impulso orgánico como de interrelación social.

Burgess (1923), siguiendo a Healy, ve al estudio de caso como una revolución metodológica ${ }^{12}$, un sustituto de los procedimientos estadísticos y de la especulación teórica. Se trata de un estudio intensivo de casos individuales y de la conducta (nótese que no se usa la expresión acción social) sobre bases empíricas e inductivas. En él no pueden aplicarse técnicas estandarizadas de medición, ya que los fenómenos de interés requieren - $\mathrm{y}$ siempre requerirán - una definición cualitativa. En el estudio de caso sociológico, según la propuesta de Burgess, se recupera la idea del sujeto en interrelación (con pares, comunidad, ambiente, etc.): el foco de atención es la "persona» (definida por oposición al individuo y, de una manera más cercana, a la idea actual de «actor») como producto de la interacción social; se refiere a alguien que

11. Esta misma tensión se evidencia en el uso de otros términos «cualitativos». Con respecto a la entrevista, por ejemplo, Cavan (1929) presenta un intento por desarrollar funciones análogas al experimento y plantea que se deben diseñar testeos para establecer las mejores formas de desarrollarla. Chapin (1924) usa el término fieldwork para referirse al trabajo de campo en el marco de la investigación estadística, hecho que, al menos en apariencia, lo distancia de un sentido exclusivamente ligado a la investigación cualitativa. Cabe aclarar que tampoco lo tiene en la actualidad, ya que con frecuencia se usa para referirse a la tarea de recolección de datos por medio de encuestas. Sin embargo, hay quienes lo usan como reemplazo de investigación cualitativa sin más (aunque, en este sentido, es más común usar la expresión field research o field study), como propone Burgess (1986).

12. House (1926) subraya con el mismo énfasis el aporte de Healy al método de los estudios de casos individuales. 
está en interacción social con sus pares, que ha adquirido estatus, es decir, una posición en la sociedad o el grupo de pertenencia, tal como postulaba Park.

Park (1930) ofrece la definición del estudio de caso más radicalmente alejada de la lógica científica canónica y, por lo tanto, más cercana a la concepción cualitativa contemporánea. Al describir la metodología propuesta por el psicólogo sueco Bjerre — que considera similar a la de Thomas y Znaniecki-, define los estudios de caso como formas de investigación que implican asociación y conversación íntima con los sujetos. Para él, esta metodología se basa en la necesidad de avanzar desde los métodos estadísticos, más generales e indirectos, superficiales e inciertos, a la observación directa, penetrando más profundamente en la realidad, la vida personal y la historia de las personas; el contacto directo y la observación personal harán avanzar la ciencia social, haciendo inteligible la realidad a partir del entendimiento (o la comprensión, ya que usa el término understanding) de los sujetos y de su conducta. En el estudio de caso científico, según sus citas de la obra de Bjerre, hay que hacer lo mismo que se hace en la vida cotidiana (observar y conversar), sólo que en la ciencia debe realizarse de modo más autoconsciente y persistente. A través de las conversaciones, o entrevistas, se buscan aquellas pronunciaciones de los sujetos en las que se evidencian lo que dan por descontado, sin jamás influenciar o inducir las respuestas. En las entrevistas que se desarrollan en el marco de un estudio de caso, hay que dejar hablar a las personas libremente e incluso estimularlas para ello. Por otra parte, no hay que tomar la «confesión» del sujeto de manera acrítica, como evidencia certera (objetiva) de algo. Las expresiones de la cara, los gestos, etc. son muy importantes, e incluso a veces más significativos que el discurso. A la objeción de que este método no es sistemático ni impersonal, es decir, que depende de la impresión individual del investigador y no se puede replicar, responde con las palabras del propio Bjerre: el científico debe basarse en lo subjetivo, pero puede recurrir a distintos tipos de fuentes y alcanzar un alto grado de objetividad ${ }^{13}$.

\section{Sobre el debate cualitativo versus el cuantitativo}

Siguiendo con la argumentación precedente, la cuestión del uso de los términos metodológicos en este período también se puede inscribir, ex post, en el marco del clásico debate cualitativo-cuantitativo. Enfatizamos el carácter retrospectivo —y, en cierto sentido, extemporáneo- de este análisis, ya que, en la época, los rótulos «métodos cualitativos» $\mathrm{y}$ «métodos cuantitativos» no eran

13. En este sentido, House (1926) plantea la existencia de un cierto consenso entre los sociólogos acerca del uso de evidencia subjetiva, pero que tal evidencia puede ser objetivamente manejada. Según él, ningún sociólogo que se autodenomine "científico» ha intentado oponerse a la idea de basar sus generalizaciones exclusivamente en información objetiva. 
corrientes y, en todo caso, no se pensaban a partir de una puja o antagonismo metodológico.

Analizar los textos a partir de este debate permite destacar dos aspectos que se han podido relevar efectivamente, y que, por otra parte, han sido señalados recurrentemente en la literatura sociológica: el uso de los términos que actualmente definimos como típicos de un enfoque cualitativo para sostener una perspectiva alternativa frente al avance de los métodos estadísticos (cuestión a la que se acaba de hacer referencia más arriba) o, por otro lado, pensar las prácticas que ellos conllevan, no de manera antagónica con los métodos estadísticos, sino en términos de complementariedad.

Lundberg (1960: 19), al analizar el desarrollo de los métodos cuantitativos en la sociología norteamericana en el período 1920-1960, indica que, en las décadas de 1920 y 1930 , se dio ya una primera gran batalla entre ambos enfoques (cualitativo y cuantitativo), pero expresada por aquel entonces a través de otros términos: métodos estadísticos y estudios de caso, respectivamente. La Escuela de Chicago (al menos hasta 1927) era generalmente considerada el exponente de los estudios de caso, mientras que la Escuela de Columbia era concebida como el bastión de los métodos cuantitativos. En esta misma línea, Alvira Martín (1983) sostiene que la primera manifestación importante del clásico enfrentamiento metodológico se produjo a partir de la publicación de la obra de Thomas y Znaniecki The Polish Peasant in Europe and America (1918).

Para Blumer (1930), esta tensión se daba en un contexto en el que la visión cada vez más hegemónica propugnaba que la ciencia social se convirtiera en una ciencia natural a través de la aplicación de procedimientos estadísticos. Dado que — según la visión dominante en la época- el éxito de las ciencias naturales devenía del carácter objetivo de sus datos y del tratamiento cuantitativo, las ciencias sociales deberían seguir ese mismo camino. Uno de los máximos exponentes de esta corriente era Lundberg, cuyo libro sobre investigación social reseña Blumer (1930). Para Lundberg, lo fundamental era la observación precisa y objetiva como primer paso del método científico; sin instrumentos estandarizados se está expuesto al sesgo y al prejuicio, por lo que se vuelve necesario desarrollar medios mecánicos de observación. En este sentido, Lundberg critica a los estudios de caso, las historias de vida y las entrevistas, porque sus datos no pueden ser tratados cuantitativamente ni generalizados, y se caracterizan por su falta de estandarización, lo que las vuelve poco útiles para la ciencia. Blumer (1930) no niega la importancia de la precisión en la observación, pero duda de que esto se logre sólo a través de instrumentos estandarizados, especialmente en un campo en el que no se sabe con absoluta certeza qué es lo que se tiene que observar. En este caso, se requiere de formas de observación que puedan redireccionarse en el marco de una perspectiva dinámica: propone entonces la búsqueda flexible bajo la guía de la imaginación sensibilizada. Que la observación deba adecuarse a los procedimientos estadísticos sólo se justificaría, para este último autor, si tales procedimientos fueran el único sinónimo posible de método científico, pero esto es muy controversial. Blumer cree que los estudios de 
caso, las historias de vida y las entrevistas pueden ser útiles porque permiten generalizaciones que no son de naturaleza estadística, y forzarlas - como plateaban muchos otros autores- dentro de los cánones tradicionales las desvirtuaría y destruiría su valor.

Con respecto a la complementariedad metodológica, una de cuyas manifestaciones actuales se conoce como triangulación, cabe destacar, en primer lugar, que los estudios supuestamente cualitativos no parecen haber sido excluyentes en la Escuela de Chicago: Forni (1993) y otros han ilustrado, en efecto, la importancia de la estadística en esta institución, y especialmente los avances que se produjeron en esta línea a partir de la llegada de Ogburn a finales de la década de 1920 .

Pero tal vez lo más interesante se relacione no tanto con la convivencia de ambos métodos en el mismo ámbito institucional, sino con su uso conjunto. En este sentido, Cavan (1929), por ejemplo, plantea que las debilidades que presenta el análisis de las historias de vida (generalmente de sentido común) pueden superarse a través del uso de la estadística. Chaffee (1930), por su parte, propone complementar los estudios de caso con historias de vida - que permiten recuperar aspectos subjetivos- y con la estadística. También Hoffer postula, en su comentario al artículo de Melvin (1927), la existencia de una pluralidad de métodos en la investigación social; pero si Chafee le asigna mayor importancia relativa al estudio de caso, que en su propuesta ocupa un lugar central frente al carácter suplementario de los otros métodos, Hoffer destaca, en cambio, la superioridad de los métodos estadísticos. Anticipando una idea que luego sería típica de la metodología canónica, sostiene que los estudios de caso sólo tienen una función exploratoria, proveyendo pistas para un posterior recurso a los más "potentes - y científicos- métodos estadísticos».

\section{Comentarios finales}

En este artículo, se ha realizado un relevamiento de algunos de los usos y sentidos asociados a los términos denotativos de los abordajes cualitativos en la Escuela de Chicago. Si bien autores como Platt (1982, 1983, 1985), Bryman (2001); Bulmer, (1984); Chapoulie (1987); Cressey (1983) y Hammersley (1989) han cuestionado su estatus cualitativo en el sentido actual, los resultados de este trabajo tienden a sugerir que esta apreciación no debería entenderse con absoluta rigidez, ya que se pueden vislumbrar en la época algunos avances incipientes en la dirección de lo que hoy se define como característico de los enfoques cualitativos.

Si bien existen pocas alusiones directas al problema del método - como lo afirma Bulmer - esto no implica la inexistencia de una preocupación metodológica y de un cuerpo definido de conocimiento especializado en proceso de formación. En este sentido, cabe destacar que el análisis de los materiales seleccionados denota un interés creciente por las cuestiones metodológicas (aunque más bien ligado al debate sobre el estatus científico de las ciencias sociales), que se refleja en la publicación cada vez más frecuente de manuales 
cuyo propósito era la sistematización del conocimiento metodológico hasta entonces disperso.

En el contexto de la discusión sobre el estatus de la sociología (y de las ciencias sociales en general) y su relación con las ciencias naturales, una preocupación claramente metodológica se presenta en el debate acerca de la pertinencia de «importar» los métodos de las ciencias naturales o la necesidad de generar otros propios. Respecto a esto último, es de hacer notar la existencia de un extendido consenso acerca de los límites de la experimentación —método característico de las ciencias naturales - en el campo de las ciencias sociales.

Asimismo, el análisis del corpus permitió identificar dos tipos de respuesta frente a estos límites: por un lado, la promoción de métodos alternativos al experimento, aunque buscando funciones equivalentes, $y$, por otro lado, la postulación de metodologías radicalmente diferentes. No obstante, las referencias a los «estudios de caso», la «observación participante», las «entrevistas», etc. (términos metodológicos que hoy tenderíamos a ligar a dichas alternativas diferentes, de naturaleza interpretativa) aparecen en algunos casos modeladas bajo los cánones clásicos de las ciencias naturales y, en este sentido, se promovía a dichas estrategias y técnicas como medios adecuados para investigar «objetivamente», generalizar, formular leyes, etc.

Ahora bien, en el marco de esta discusión, y como muestra de ciertos avances incipientes en la dirección de los enfoques cualitativos contemporáneos, resulta especialmente relevante el artículo de Park (1930), quien define el estudio de caso como una forma de investigación que implica asociación y conversación íntima con los sujetos, con lo cual relativiza, además, el lugar central de la objetividad.

También es de destacar la preocupación de algunos investigadores por la complementariedad de los métodos utilizados en la investigación empírica. En efecto, para muchos de ellos, las distintas propuestas metodológicas no representaban modelos antagónicos, sino complementarios, principalmente los estudios de caso y la estadística.

Finalmente, cabe señalar que, en un momento en que los métodos estadísticos eran propuestos por muchos sociólogos como superiores — desde el punto de vista de su cientificidad—, comenzaban también a surgir voces críticas, especialmente entre los autores de la Escuela de Chicago. Es en los textos de Park, Burgess, House y Blumer en los que se encuentran, aunque aún de manera embrionaria, argumentaciones epistemológico-metodológicas más distanciadas de las visiones canónicas (y de las de colegas pertenecientes a otros ámbitos institucionales en los que se defendían a los métodos hoy llamados «cualitativos» en los términos de la ciencia tradicional). Está claro, sin embargo, que la propuesta "cualitativista» de los chicaguenses no puede explicarse a partir de fundamentos teóricos interpretativos centrados en el concepto weberiano de verstehen — como bien ha indicado Platt (1985)—, y muchos menos de carácter fenomenológico, es decir, las corrientes teóricas que más han marcado el desarrollo contemporáneo de los métodos cualitativos. 


\section{Referencias bibliográficas}

Alvira MarTín, F. (1983). «Perspectiva cualitativa-perspectiva cuantitativa en la metodología sociológica». REIS: Revista Española de Investigaciones Sociológicas, 22, 5375.

BeCKer, H. (1999). «The Chicago School, So-Called». Qualitative Sociology, 22, (1), 3-12.

BRYMAN, A. (2001). Ethnography. Londres: Sage.

Bulmer, M. (1984). The Chicago School of Sociology: Institutionalization, Diversity, and the Rise of Sociological Research. Chicago: University Press.

Burgess, R. (1986). In the field: An introduction to field research. Londres: Routledge.

Chapoulie, J. (1987). «Everett C. Hughes and the Development of Fieldwork in Sociology». Urban Life, 15 (3), 259-298.

Cortese, A. (1995). "The Rise, Hegemony, and Decline of the Chicago School of Sociology». Social Science Journal, 32 (3), 235-254.

CREssey, D. (1983). "The methodology of the taxi-dance hall: an early account of Chicago ethnography from de 1920s». Urban Life, 12, 109-19.

Denzin, N. y LinCOLn, Y. (1994). The Sage handbook of Qualitative Research. Thousands Oaks: Sage.

FORNI, F. (1993). «Estrategias de recolección y estrategias de análisis en la investigación social». En: FORNI, F. et al. Métodos cualitativos II: La práctica de la investigación. Buenos Aires: CEAL.

GILMORE, S. (1988). "Schools of Activity and Innovation». Sociological Quarterly, 29, 203-219.

Hammersley, M. (1989). The dilemma of qualitative method. Herbert Blumer and the Chicago Tradition. Londres / Nueva York: Routledge.

LundBerG, G. (1960). «Quantitative Methods in Sociology: 1920-1960». Social Forces, 39 (1), 19-24.

Marradi, A.; Archenti, N. y Piovani, J.I. (2007). Metodología de las ciencias sociales. Buenos Aires: Emecé.

PlatT, J. (1982). «The Origin Myth of Participant Observation». American Sociological Association.

- (1983). "The Development of the "Participant Observation" Method in Sociology: Origin Myth and History». Journal of the History of the Behavioral Sciences, 19 (4), 379-393.

- (1985). "Weber's Verstehen and the History of Qualitative Research: The Missing Link». British Journal of Sociology, 36 (3), 448-466.

- (1996). A History of Sociological Research Methods in America. Cambridge: University Press.

TAYLOR, S.J. y BOGDAN, R (1986). Introducción a los métodos cualitativos de investigación. Barcelona: Paidós.

Thomas, W.I. y ZnAniecki, F. (1918). The Polish Peasant in Europe and America: Chicago: University Press.

VASILACHIS, I. (1993). Métodos cualitativos I. Los problemas teórico-epistemológicos. Buenos Aires: CEAL. 


\section{Anexo}

Listado de fuentes primarias citadas:

Blumer, H. (1930). Book Review (Social Research. George A. Lundberg; Research in the Social Sciences. Wilson Gee; An Introduction to Social Research. Howard W. Odum, Katharine Jocher). American Journal of Sociology, 35 (6), 1101-1111.

BOUCKE, O.F. (1923). «The limits of Social Science II». American Journal of Sociology, 28 (4), 443-460.

BuRgESS E.W. (1923). "The study of the delinquent as a person». American Journal of Sociology, 28 (6), 657-680.

- (1923). Book Review (Judge Baker Foundation Case Studies. William Healy, Augusta F. Bronner; The Kingdom of Evils: Psychiatric Social Work Presented in One Hundred Case Histories Together with a Classification of Social Division of Evil. E. E. Southard, Mary C. Jarrett; The Defective Delinquent and Insane. Henry A. Cotton; Crime: Its Cause and Treatment. Clarence Darrow; Juvenile Deliquency. Henry H. Goddard). American Journal of Sociology, 28 (5), 618-621.

- (1926). «Topical summaries of current literature: The Family». American Journal of Sociology, 32 (1), 104-115.

CAVAN, R.S. (1929). «Topical summaries of current literature: interviewing for lifehistory material». American Journal of Sociology, 35 (1), 100-115.

CHAFFEE, G.E. (1930). «The isolated religious sect as an object for social research». American Journal of Sociology, 35 (4), 618-630.

CHAPIN, F.S. (1924). «The statistical definition of a societal variable». American Journal of Sociology, 30 (2), 154-171.

HolT, A.E. (1926). «Case records as Data for Studying the conditioning of religious experience by social factors». American Journal of Sociology, 32 (2), 227-236.

HousE, F.N. (1926). "Topical Summaries of Current Literature: The Logic of Sociology». American Journal of Sociology, 32 (2), 271-287.

Kunlaman, A.F. (1930). Book Review (The Technique of Research in Education. Claude C. Crawford). American Journal of Sociology, 35 (4), 657-659.

Melvin, B.L. (1927). «Methods of social research». American Journal of Sociology, 33 (2), 194-210.

NELSON, R.W. (1924). "The logical method of science». American Journal of Sociology, 29 (5), 553-570.

PARK, R.E. (1923). Book Review (An Introduction to Reflective Thinking. Laurence Buermeyer, William Forbes Cooley, John J. Coss, Horace L. Friess, James Gutmann, Thomas Munro, Houston Peterson, John H. Randall, Jr., Herbert W. Schneider). American Journal of Sociology, 29 (3), 364-365.

- (1930). «Murder and the case study method». The American Journal of Sociology, 36 (3), 447-454.

QueEN, S.A. (1929). Book Review (Field Studies in Sociology: A Student's Manual. Vivien M. Palmer). American Journal of Sociology, 34 (6), 1197-1198.

THOMAS, D.S. (1929). «Statistics in social research». American Journal of Sociology, 35 (1), 1-17.

WoOD, A.E. (1927). «A program for Criminological Research». American Journal of Sociology, 33 (3), 431-443. 